\title{
Two Hybrid Metaheuristic Algorithms for Hot Rolling Scheduling
}

\author{
Lixin TANG, ${ }^{1)}$ Xiaoxia ZHANG ${ }^{21}$ and Qingxin GUO ${ }^{11}$ \\ 1) Liaoning Key Laboratory of Manufacturing System and Logistics, The Logistics Institute, Northeastern University, \\ Shenyang, China. $\quad$ 2) The Software College, University of Science and Technology Liaoning, China. \\ E-mail: qhjytlx@mail.neu.edu.cn, syzhangxx@163.com,guoqingxin@ise.neu.edu.cn
}

(Received on May 26, 2008; accepted on January 16, 2009)

\begin{abstract}
This paper presents a model for the hot rolling scheduling problem (HRSP), which is derived from the actual steel production. The model is characterized by some new features, such as the rolling length of the consecutive slabs with the same width, temperature jump between adjacent slabs. The warm up part is a minor part of a turn and is usually ignored, but it affects directly the product quality. Therefore, besides the slab sequence in the staple material section, we also consider the slab sequence in the warm up material section. These features make the solution methodology more difficult. Therefore, two hybrid strategies are proposed to determine good approximate solutions for this complicated problem. The first one (CT_ACO) is a hybrid strategy based on the solution construction mechanism of ant colony optimization (ACO) with cyclic transfers (CT). The second one (CT_SS) is to hybridize scatter search (SS) and CT neighborhood search. Moreover, we design a decision support system in which two algorithms have been embedded for the HRSP. The most popular feature of the system is the architecture of component management, which allows us to modify easily some components according to the practice situation. The computational experiments show that CT_ACO is superior to general ACO, and CT_SS is also better than SS in terms of solution quality. The CT_ACO method and CT_SS have more potential for improvement to solve the HRSP compared with the current scheduling method, and the CT_ACO generates slightly better quality solutions than CT_SS algorithm.
\end{abstract}

KEY WORDS: cyclic transfer; ant colony optimization; a hybrid search algorithm; scatter search; hot rolling scheduling; the decision support system.

\section{Introduction}

The modern iron and steel industries are beginning to give more serious attention to the theoretical and technical methods that promise to increase steel production efficiency and make the best of their existing capacities to ensure their survival in the global market. In this paper we focus our attention on the hot strip mill area. The hot strip mill is often regarded as the bottleneck of the overall production process while the slab sequence is the key to the HRSP. In the hot strip mill, a typical problem is how to make the rolling scheduler, which plays an important role not only in the hot strip mill, but also in the integrated production of continuous casting and hot-charging rolling. Most steel production facilities produce a variety of finished products according to customer orders. Each order requires the production of a number of coils with the physical properties and dimensions, and each coil corresponds to one slab. Steel production involves a range of processes to produce such coils. Raw materials are transformed by a series of chemical reactions in the blast furnace into liquid iron. ${ }^{1)}$ Next, the liquid iron is transferred into liquid steel through chemistry and purification processes in the melt shop. After the chemistry requirements have been met, the liquid steel is transformed into solid large steel bars in the continuous caster process, and in the hot strip mill into the final product, steel coils.
The hot strip mill transfers steel slabs into steel coils by subjecting the slabs first to high temperatures in a furnace and then to high pressures through a series of rolls. In hot strip mill production, the roller performance plays a significant role in determining product quality and setup cost. Roller replacements involve a very high setup cost, so we wish to replace rollers as infrequently as possible, ${ }^{2,3)}$ while still maintaining the high coil quality.

We investigate the HRSP for the actual steel production. The current scheduling system uses a serial strategy and heavily relies on skilled human experts. Therefore the scheduling horizon was limited to their experiences and abilities. In order to utilize the automated scheduler more efficiently, we adopted a parallel strategy here to simultaneously group the slabs into multiple turns for a whole shift and sequence the slabs in each turn so as to achieve global optimization. The objective is to find the effective product scheduling that minimizes the costs of roller replacement, total changeover costs between adjacent slabs, and the penalties of the unrolled slabs paid and warm up material slabs used in the staple material section. Total changeover costs between adjacent slabs and the costs of roller replacement are two obvious conflicting objectives. Minimizing total changeover costs can be obtained by improving product quality. Each slab has several characteristics: width, hardness, gauge, etc. The greater the characteristic differ- 
ence between adjacent slabs, the greater the impact on the roller. To avoid marks in the coils and guarantee product quality, the most important restrictions require that changes in width, hardness and gauge should be gradual. Improving product quality requires changing the rollers as frequently as possible, whereas minimizing the costs of roller replacement requires that a work roller should roll as many slabs as possible in a turn. Therefore, there are some tradeoffs between these objectives and they will be obtained by the effective hot rolling scheduling.

The HRSP is an important research topic and has been widely explored recently. Balas (1989) $)^{4)}$ and Lopez et al. ${ }^{1)}$ present a prize collecting traveling salesman problem (PCTSP). However, they achieved the solution with such a serial strategy, which suffers from the disadvantage of local optimization. Tang et al. ${ }^{5)}$ modeled the HRSP as a multiple traveling salesman problem based on actual production constraints. Using the proposed parallel strategy, they scheduled simultaneously multiple turns for the whole shift from a global optimal view. However, they did not take into account the capacity constraint. Cowling ${ }^{2}$ presented a prize collecting vehicle routing problem (PCVRP) model. A heuristic based on local search and tabu search techniques was presented. The heuristic proposed gave better results than the existing manual system. Cowling ${ }^{6)}$ described a flexible decision support system for the HRSP. In that paper, they concentrated on the implementation of the system in practice, while we focus not only on the implementation of the system, but also on the detailed model and solution method.

The main contribution of this paper differs from the papers described above on following points: First, this paper presents the detailed model with multiple objectives and practical constraints to make the hot rolling scheduling more efficiently. Many factors and constraints such as the rolling length of the consecutive slabs with the same width, temperature jump between adjacent slabs and the capacity limits of turns should be taken into account. Second, when a turn is arranged, the warm up part is a minor part of the turn and usually ignored. Therefore, besides the slab sequence in the staple material section, we also consider the slab sequence in the warm up material section. Moreover, since the mathematical model is more complicated, two hybrid algorithms are proposed to determine good approximate solutions for this complicated problem. Finally, a decision support system for the HRSP is designed to improve the manual scheduling process. The remainder of this paper is organized as follows. Section 2 introduces constraints and model formulation. Solution methodology and two hybrid algorithms are described in Sec. 3. Section 4 gives the details of decision support system construction. Section 5 provides the computational experiments comparing two algorithms using various scheduling scenarios. Finally, Sec. 6 presents some conclusions.

\section{Constraints and Model Formulation}

\subsection{Hot Rolling Scheduling Constraints}

The hot rolling mill transfers the slabs into steel coils by subjecting to high pressures through a series of rolls, which are divided mainly into work rolls and backup rolls in the hot strip mill. Work rolls are in direct contact with the hot steel strand, and backup rolls exert pressure on the work rolls. Because of suffering the high temperature, high speed, heavy wear and tear, work rollers and backup rollers on each stand often need to be replaced at regular intervals. In the hot rolling plant, rolling objects between two work rollers are called a hot rolling turn, which is basic units of hot rolling. A hot rolling set is made up of multiple hot rolling turns. So rolling objects are organized into turns and then several turns are arranged into a shift. Every workday is often divided into three shifts. Schedulers do hot rolling scheduling for the next shift during the current shift. Figure 1 shows the composition of a hot rolling schedule for a shift where $M$ denotes the number of turns. The most important constraints require smooth changes in dimension, temperature and hardness between adjacent slabs. These constraints affect directly the product quality and roller wear. Smooth changes in the width of the slabs form a particular profile of coffin shape in a turn (see Fig. 1). In a turn, there are the warm up material section, in which the slabs should be scheduled from narrow to wide, and the staple material section, in which the slabs should be scheduled from wide to narrow. In the warm up material section, if slab $j$ is rolled immediately after slab $i$ in the same turn, difference in the width is $w_{i}-w_{j}$. If $w_{i}-w_{j}<0$, the difference should be considered as normal jump. Otherwise, it denotes abnormal jump. In the staple material section, if slab $j$ is rolled immediately after slab $i$ in the same turn, difference in the width is $w_{i}-w_{j}$. If $w_{i}-w_{j}>0$, the difference should be regarded as normal jump. Otherwise, it represents abnormal jump.

Because the HRSP with practical constraints is extremely complex, besides common constraints such as smooth changes in dimension and hardness between adjacent slabs, some special constraints such as the rolling length of the consecutive slabs with same width, temperature jump be-

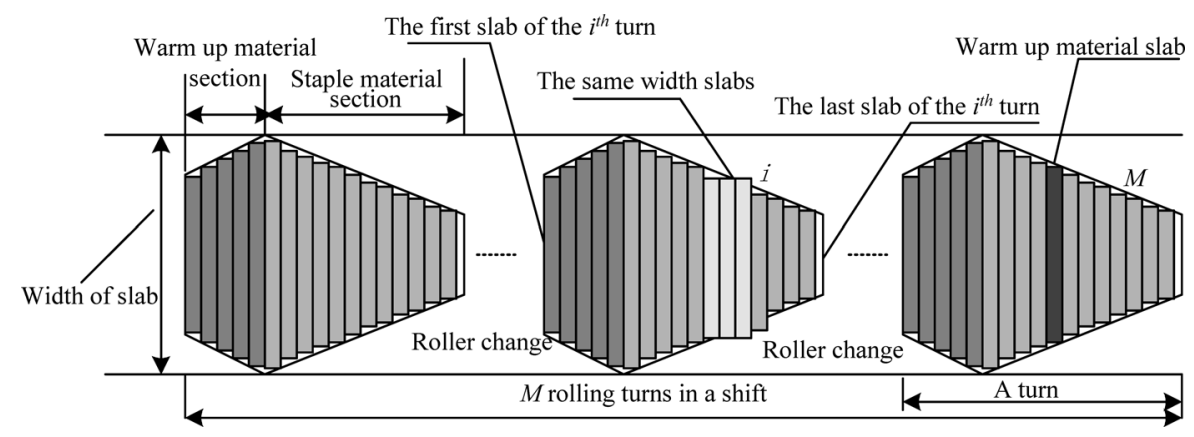

Fig. 1. The composition of a hot rolling schedule for a shift. 
tween adjacent slabs, the capacity limits of turns, etc. as compared with the papers in the literature described above should be considered in our mathematical model. The objective is to find an optimal set of turns through minimizing a cost function consisting of the following terms. 1) Minimizing total changeover costs to ensure product quality. 2) The high costs of roller replacement to require as many rolled slabs as possible in a turn. 3) The penalties paid due to slabs that are not rolled. 4) Minimizing penalties paid for warm up material slabs used in the staple material section to make these slabs as fewer as possible.

\subsection{Model Formulation}

The following symbols are used for defining the problem parameters and variables.

Parameters:

$N$ : The set of all slabs, $N=\{1,2, \ldots, n\} . N^{\prime}=\{0,1,2$, $\ldots, n\}$.

$\gamma_{i}$ : The penalty that slab $i$ will cause when it is unrolled.

$d_{i}$ : The length of slab $i$.

$M$ : The set of all turns, $M=\{1,2, \ldots, m\}$, where $m$ represents the total number of turns a shift.

$l$ : The type of section, $l \in\{1,2\}$, where 1 denotes the staple material section and 2 denotes the warm up material section.

$Q^{l}$ : The maximum capacity of section $l$ each turn can roll.

$O^{l}$ : The minimum capacity of section $l$ each turn can roll.

$R^{1}$ : The rolling length of consecutive slabs with same width in the staple material section of a turn.

$V$ : The fixed cost of a turn.

$A_{1}$ : The set of candidate slabs chosen to be in the staple material section.

$A_{2}$ : The set of candidate slabs selected to be in the warm up material section and $A_{1} \cup A_{2}=N$.

$\delta_{1}^{l}$ : The maximum changes in width between adjacent slabs, where $\delta_{1}^{1}$ denotes normal jump limits of width in the staple material section, and $\delta_{1}^{2}$ represent abnormal jump limits of width in the warm up material section.

$\delta_{2}^{l}$ : The maximum changes in width between adjacent slabs, where $\delta_{2}^{1}$ denotes abnormal jump limits of width in the staple material section, and $\delta_{2}^{2}$ represent normal jump limits of width in the warm up material section.

$\varphi_{1}^{1}$ : The gauge parameter of the next slab if abnormal jumps in width between adjacent slabs happen in staple material sections.

$\varphi_{2}^{1}$ : The hard parameter of the next slab if abnormal jumps in width between adjacent slabs happen in staple material sections.

$V_{m}:$ A very large number.

$\rho^{l}$ : The jump limits in hardness between adjacent slabs in the section $l$.

$l^{\prime}$ : The type of slab temperature, $l^{\prime} \in\{1,2,3\}$, where $l^{\prime}=1,2$ and 3 denote output temperature from heating furnace, temperature of rolling end and curly temperature respectively.

$\psi_{i^{\prime}}^{l}$ : The jump limits in $i^{\prime}$ type of slab temperature between adjacent slabs in the section $l$. $\vartheta^{l}:$ The jump limits in gauge between adjacent slabs in the section $l$.

$\varpi$ : The task efficiency parameter.

$\eta$ : The maximum number of warm up material slabs used in staple material sections.

$B$ : The cost of warm up material slabs used in staple material sections.

$w_{i}$ : The width of slab $i$.

$h_{i}$ : The hardness of slab $i$.

$g_{i}$ : The gauge of slab $i$.

$t_{i}^{l^{\prime}}$ : The temperature of slab $i$ in $i^{\prime}$ type of temperature.

$C_{i j}^{l}$ : Penalties for the changes in the section $l$ when scheduling slab $j$ directly after slab $i$. When $i=j$, $C_{i j}^{l}=\infty, C_{i j}^{l}=p_{i j}^{l w}+p_{i j}^{l g}+p_{i j}^{l h}+p_{i j}^{l t}$, where, $p_{i j}^{l w}, p_{i j}^{l g}, p_{i j}^{l h}$ and $p_{i j}^{l t}$ represent penalty for width, gauge, hardness and temperature jumps from slab $i$ to slab $j$ respectively.

$$
p_{i j}^{l w}= \begin{cases}0, & \text { if slabs } i \text { and } j \text { have the same width } \\ \omega_{1}^{l}, & \text { if } 0<w_{i}-w_{j} \leq \delta_{1}^{l} \\ \omega_{2}^{l}, & \text { if } 0<w_{j}-w_{i} \leq \delta_{2}^{l} \\ \infty, & \text { otherwise }\end{cases}
$$$$
p_{i j}^{l g}= \begin{cases}0, & \text { if slabs } i \text { and } j \text { have the same gauge } \\ \omega_{3}^{l}, & \text { if } 0<\left|g_{i}-g_{j}\right| \leq \vartheta^{l} \\ \infty, & \text { otherwise }\end{cases}
$$$$
p_{i j}^{l h}= \begin{cases}0, & \text { if slabs } i \text { and } j \text { have the same hardness } \\ \omega_{4}^{l}, & \text { if } 0<\left|h_{i}-h_{j}\right| \leq \rho^{l} \\ \infty, & \text { otherwise }\end{cases}
$$
$p_{i j}^{l t}=\sum_{i^{\prime}=1}^{3} \mu_{i j}^{l i^{\prime}}, \quad \begin{aligned} & \text { where } \mu_{i j}^{l i^{\prime}} \text { is penalty for } i^{\prime} \text { type of temperature } \\ & \text { jumps from slab } i \text { to slab } j\end{aligned}$

$$
\mu_{i j}^{l i^{\prime}}= \begin{cases}0, & \text { if slabs } i \text { and } j \text { have the same values of } i^{\prime} \text { type } \\ & \text { temperature } \\ \varepsilon_{i^{\prime}}^{l}, & \text { if } 0<\left|t_{i}^{i^{\prime}}-t_{j}^{i^{\prime}}\right| \leq \psi_{i^{\prime}}^{l} \\ \infty, & \text { otherwise }\end{cases}
$$

where $\omega_{1}^{l}, \omega_{2}^{l}, \omega_{3}^{l}, \omega_{4}^{l}$ and $\varepsilon_{i^{\prime}}^{l}$ are the penalties.

Decision variables:

$x_{i j k}^{1}= \begin{cases}1 & \begin{array}{l}\text { if turn } k \text { rolls slab } j \text { directly after slab } i \text { and } i \neq j \\ \text { in the staple material section }\end{array} \\ 0 & \text { otherwie }\end{cases}$

$x_{i j k}^{2}= \begin{cases}1 \quad & \text { if turn } k \text { rolls slab } j \text { directly after slab } i \text { and } i \neq j \\ & \text { in the warm up material section } \\ 0 & \text { otherwie }\end{cases}$

$y_{i k}=\left\{\begin{array}{ll}1 & \text { if slab } i \text { is rolled by turn } k \\ 0 & \text { otherwise }\end{array} \quad i \in N ; k \in M\right.$ 
Objective:

$$
\begin{aligned}
\operatorname{Min} . \lambda_{1} \sum_{k=1}^{m} & \sum_{i=0}^{n} \sum_{j=0}^{n}\left(C_{i j}^{1} x_{i j k}^{1}+C_{i j}^{2} x_{i j k}^{2}\right)+\lambda_{2} \sum_{k=1}^{m} \sum_{i=1}^{n} V x_{0 i k}^{2} \\
& +\lambda_{3} \sum_{k=1}^{m} \sum_{i=1}^{n} \gamma_{i}\left(1-y_{i k}\right) \\
& +\lambda_{4} B \sum_{k=1}^{m}\left(\sum_{i \in A_{2}} \sum_{j \in A_{1}} x_{i j k}^{1}+\sum_{i \in N} \sum_{j \in A_{2}} x_{i j k}^{1}\right) \ldots \ldots . .
\end{aligned}
$$

Subject to

$$
\begin{aligned}
& \sum_{k=1}^{m} y_{i k} \leq 1, \quad i \in N \\
& \sum_{k=1}^{m} \sum_{i=1}^{n}\left(x_{0 i k}^{1}+x_{0 i k}^{2}\right)=\sum_{k=1}^{m} \sum_{i=1}^{n}\left(x_{i 0 k}^{1}+x_{i 0 k}^{2}\right) \\
& O^{l} \leq \sum_{i=1}^{n} d_{i} \sum_{j=1}^{n} x_{i j k}^{l} \leq Q^{l}, \quad k \in M ; l=1,2 \\
& \sum_{k=1}^{m} \sum_{i=1}^{n} \sum_{j=1}^{n}\left(x_{i j k}^{1}+x_{i j k}^{2}\right) / n \geq \varpi
\end{aligned}
$$

The rolling length restrictions on the consecutive slabs with the same width

$$
\begin{aligned}
& \sum_{j=1, j \neq i}^{n}\left(x_{i j k}^{1}+x_{i j k}^{2}\right)=\sum_{j=1, j \neq i}^{n}\left(x_{j i k}^{1}+x_{j i k}^{2}\right)=y_{i k}, \quad i \in N ; k \in M \\
& w_{i}-w_{j} \leq \delta_{1}^{l}+\left(1-x_{i j k}^{l}\right) V_{m}, \quad i, j \in N ; l=1,2 ; k \in M \\
& w_{j}-w_{i} \leq \delta_{2}^{l}+\left(1-x_{i j k}^{l}\right) V_{m}, \quad i, j \in N ; l=1,2 ; k \in M \text {. } \\
& g_{j} \geq \varphi_{1}^{1}-V_{m}\left(1-x_{i j k}^{1}\right), \quad i, j \in N ; k \in M ; w_{j}<w_{i} \\
& h_{j} \leq \varphi_{2}^{1}+V_{m}\left(1-x_{i j k}^{1}\right), \quad i, j \in N ; k \in M ; w_{j}<w_{i} \\
& \left|h_{j}-h_{i}\right| \leq \rho^{l}+\left(1-x_{i j k}^{l}\right) V_{m}, \quad i, j \in N ; l=1,2 ; k \in M \\
& \left|t_{j}^{l^{\prime}}-t_{i}^{l^{\prime}}\right| \leq \psi_{i^{\prime}}^{l}+\left(1-x_{i j k}^{l}\right) V_{m}, \\
& i, j \in N ; l=1,2 ; l^{\prime}=1,2,3 ; k \in M \\
& \left|g_{i}-g_{j}\right| \leq \vartheta^{l}+\left(1-x_{i j k}^{l}\right) V_{m}, i, j \in N ; l=1,2 ; k \in M \ldots \\
& \sum_{i, j \in \Omega}\left(x_{i j k}^{1}+x_{i j k}^{2}\right) \leq|S|-1, \quad S \subset N, 2 \leq|S|<n ; k \in M \\
& x_{i j k}^{1}+x_{i j k}^{2} \leq 1, \quad i, j \in N, k \in M \\
& \sum_{k=1}^{m}\left(\sum_{i \in A_{2}} \sum_{j \in N} x_{i j k}^{1}+\sum_{i \in A_{1}} \sum_{j \in A_{2}} x_{i j k}^{1}\right) \leq \eta
\end{aligned}
$$

$x_{i j k}^{2} \in\{0,1\}, \quad i, j \in A_{2}, k \in M$

$y_{i k} \in\{0,1\}, \quad i \in N, k \in M$

The objective function (1) consists of the minimization of the costs of total changeover costs between adjacent slabs, of roller replacement, of the penalties to be paid for the unrolled slabs and of the penalties to be paid for warm up material slabs used in the staple material section. $\lambda_{1}, \lambda_{2}, \lambda_{3}, \lambda_{4}{ }^{\prime} \in[0,1]$ are four preset coefficients such that $\lambda_{1}+\lambda_{2}+\lambda_{3}+\lambda_{4}=1$. Constraints (2) ensure that a slab can only be rolled one time at most. Constraints (3) guarantee that each turn starting the virtual node must return to it in the end. The virtual node is introduced into the HRSP and all turns start from and end at this virtual node to make closed turns. The Eqs. (4) ensure the capacity constraints of turns in the warm up material section and the staple material section. Constraints (5) ensure that the percentage of rolled slabs should be no less than a predetermined amount. Constraints (6) require that the rolling length of the consecutive slabs with the same width should never exceed the predetermined limits in one turn (see Fig. 1). Constraints (7) ensure that when a slab is rolled in a certain turn, there should be exactly one slab before and one slab after it in the turn respectively. The Eqs. (8) and (9) represent the constraints of normal changes and abnormal changes of the adjacent slab width in the staple material section and in the warm up material section respectively. The Eqs. (10) and (11) are the constraints of slabs in gauge and hardness when abnormal changes of the adjacent slabs in width happen in the staple material section. The Eqs. (12), (13) and (14) represent the constraints of smooth changes of the adjacent slabs in hardness, temperature and gauge. Constraints (15) avoid subschedules for in the feasible solutions. Without Constraints (15), the subschedules would be a feasible solution, but they are not a complete schedule in the solution. For further information on this type of constraints, please refer to Ref. 5). Constraints (16) guarantee that the slab is used one time at most in warm up material or in the staple material section. Constraints (17) ensure that the number of warm up material slabs used in the staple material section cannot exceed a predetermined amount. Red color slab denotes the warm up material slab used in the staple material section (see Fig. 1). On the contrary, the staple material slabs cannot be used in the warm up material section. Constraints (18), (19) and (20) specify the integrity conditions on the variables.

Although the HRSP has been studied in some literatures, little work has been done on the detailed model accounting for many practical constraints. Existing models are either special cases of our model or have a different structure. Our model differs from the literature described above in four aspects. First, besides the slab sequence in the staple material section, we also consider the slab sequence in the warm up material section. Second, our model takes into account the rolling length of the consecutive slabs with the same width, temperature jumps between adjacent slabs, and the capacity limits of turns, etc. Third, not every slab must be rolled compulsorily but a penalty can be collected from the unrolled slab. Finally, we make simultaneously multiple turns for a whole shift. 


\section{Solution Methodology}

In the literature described above, some researchers formulated the HRSP as TSP or VRP model, which is an NPhard problem. Our model is derived from the actual hot rolling production and many practical constraints must be taken into account, so these make our model more complicated. Some current metaheuristic methods are effective to solve the complicated problem and can be used to obtain high quality solutions, therefore, two hybrid CT_ACO and CT_SS algorithms are proposed to determine good approximate solutions. Due to its exploration and information learning abilities, ACO is expected to provide an appropriate initial schedule without difficulty. SS considers both solution quality and diversification, and it is very flexible and effective. CT method is an enhancement and it is embedded into the $\mathrm{ACO}$ and SS procedures to improve the solutions. We will briefly describe these methods.

\subsection{Cyclic Transfers}

\subsubsection{Cyclic Transfer Neighborhoods}

The cyclic transfer $(\mathrm{CT})^{7,8)}$ is a new local search algorithm based on the multi-exchange neighborhood structure developed by Thompson and Orlin. The central concept behind cyclic transfers, as applied to the HRSP, is the attempt to improve the total cost of turns by transferring small slabs among turns in a cyclic manner. Let $I=\left\{I^{1}, I^{2}, \Lambda, I^{m}\right\}$ be a feasible solution to the HRSP and $\rho$ be a cyclic permutation of a subset of $\{1, \Lambda, m\}$. Figure 2 shows a 3 -cyclic transfer for the HRSP, with $A_{i}^{1}, A_{j}^{1} \in I^{1}, A_{i}^{2}, A_{j}^{2} \in I^{2}, A_{i}^{3}, A_{j}^{3} \in I^{3}$, and $\rho=\left(\begin{array}{ll}123 \\ 2\end{array}\right)$. The slabs of the cyclic transfer moves respectively in its section. This cyclic transfer simultaneously moves slab $A_{i}^{1}$ from $I^{1}$ to $I^{2}$, slab $A_{i}^{2}$ from $I^{2}$ to $I^{3}$, slab $A_{i}^{3}$ from $I^{3}$ to $I^{1}$ in the warm up material section, and in the same way, slab $A_{j}^{1}$ from $I^{1}$ to $I^{2}$, slab $A_{j}^{2}$ from $I^{2}$ to $I^{3}$, and slab $A_{j}^{3}$ from $I^{3}$ to $I^{1}$ in the staple material section. If $I^{\prime}$ denote the obtained solution, then cost of the exchange is $F\left(I^{\prime}\right)-F(I)$, and there is an improvement cyclic transfer if $F\left(I^{\prime}\right)-F(I)<0$. The improvement moves in terms of negative cost cycles in an associated graph are called the improvement graph.

The CT neighborhood considers solutions obtained by si-

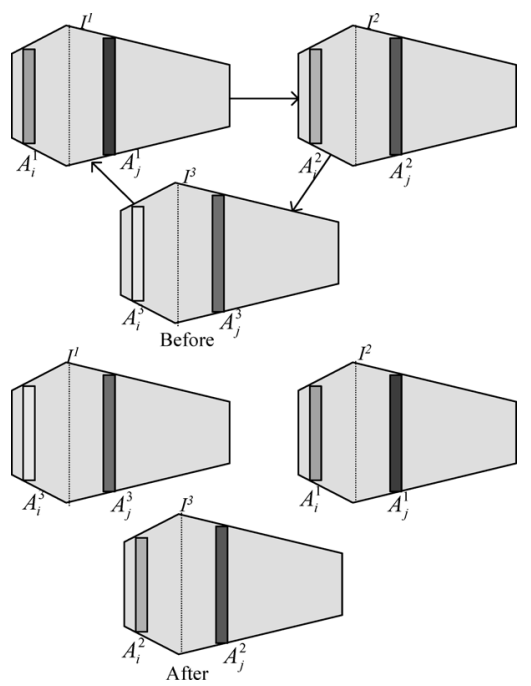

Fig. 2. The effect of a 3-cycle transfer. multaneously transferring single elements among subsets, and the CT neighborhood grows exponentially with the problem size. Moreover, each arc cost computation is itself difficult. These factors cause CT neighborhood search to be inherently more complicated, so it is unlikely that one can search the neighborhood completely in polynomial time. We should use a heuristic to approximate the neighborhood search. The heuristic is based on approximately dynamic programming to search for an improvement neighbor in the restricted CT neighborhood by limiting the length of the cycles. In order to construct a cyclic transfer, the improvement graph related to $I$ is the auxiliary graph $G=(V, E, C)$ defined as:

$V=V^{1} \cup V^{2} \cup \Lambda V^{m}$, where,

$V^{k}=\{$ sets of slabs in the same turn $k, k=1,2, \Lambda, m\}$

$E=E^{1} \cup E^{2} \cup \Lambda E^{m}$, where,

$E^{k}=\left\{(i, j): i \in V^{k}, j \in V^{k+1}, k=1,2, \Lambda, m-1\right\}$

$C=C^{1} \cup C^{2} \cup \Lambda C^{m}$, where,

$C^{k}=\left\{c(i, j):(i, j) \in E^{K}, k=1,2, \Lambda, m\right\}$ are edge cost set.

Let $I(i)$ and $I(j)$ be the different turns, in which slabs $i \in I(i)$ and $j \in I(j)$. The cost $c(i, j)$ of each transfer $(i, j)$ is defined as the cost variation of turn $I(j)$, due to simultaneously adding slab $i$ to $I(j)$ and removing slab $j$ from $I(j)$, i.e., $c(i, j)=F(I(j)+i-j)-F(I(j))$.

Given a feasible solution $I=\left\{I^{1}, I^{2}, \ldots, I^{m}\right\}$, with, $I^{1}=\left\{A_{1}^{1}, A_{2}^{1}, \Lambda, A_{N_{1}}^{1}\right\}, I^{2}=\left\{A_{1}^{2}, A_{2}^{2}, \Lambda, A_{N_{2}}^{2}\right\}, \ldots, I^{m}=\left\{A_{1}^{m}, A_{2}^{m}\right.$, $\left.\Lambda, A_{N_{m}}^{m}\right\}$, and a permutation $\rho=(\rho(1), \rho(2), \ldots, \rho(m)) . N_{m}$ is the slab number of the set $I^{m}$ and $m$ is the number of turns. Let $f_{k}\left(A_{2}^{1}, A_{j}^{k+1}\right)$ be the cost of the best transfer from the node $A_{2}^{1}$ in turn $\rho(1)$ to every node $j$ in turn $\rho(k+1)$. The iteration relationship of the best cost of transfers is:

$$
\begin{array}{r}
f_{1}\left(A_{2}^{1}, A_{j}^{2}\right)=c\left(A_{2}^{1}, A_{j}^{2}\right), \quad j=1, \cdots, N_{2} \cdots \cdots \cdots \cdots \cdots \cdots . . . \\
f_{k}\left(A_{2}^{1}, A_{j}^{k+1}\right)=\min _{1 \leq i \leq N_{k}}\left\{c\left(A_{i}^{k}, A_{j}^{k+1}\right)+f_{k-1}\left(A_{2}^{1}, A_{i}^{k}\right)\right\}, \\
j=1, \cdots, N_{k+1}, k=2, \cdots, m \ldots \ldots . .
\end{array}
$$

If $k$ is equal to the number of turns $m$, let $I^{m+1}=I^{1}$. If $f_{k}\left(A_{2}^{1}, A_{j}^{k+1}\right)<0$, an improvement cyclic transfer with the improved objective value is found. An example of node $A_{2}^{1}$ $\left(A_{2}^{1} \in I^{1}\right)$ the search proceeding for approximately dynamic programming is shown in Fig. 3, in which all possible transfers are given, and the permutation $\rho=\{1,2,3\}$. Transfers $\left(A_{2}^{1}, A_{3}^{2}\right),\left(A_{3}^{2}, A_{2}^{3}\right)$ and $\left(A_{2}^{3}, A_{2}^{1}\right)$ can form a negative cost cycle $\left\{A_{2}^{1}-A_{3}^{2}-A_{2}^{3}-A_{2}^{1}\right\}$. From this, we can see that some transfers such as $\left(A_{2}^{1}, A_{1}^{2}\right),\left(A_{1}^{2}, A_{1}^{3}\right)$ and $\left(A_{1}^{3}, A_{1}^{1}\right)$ can cause the

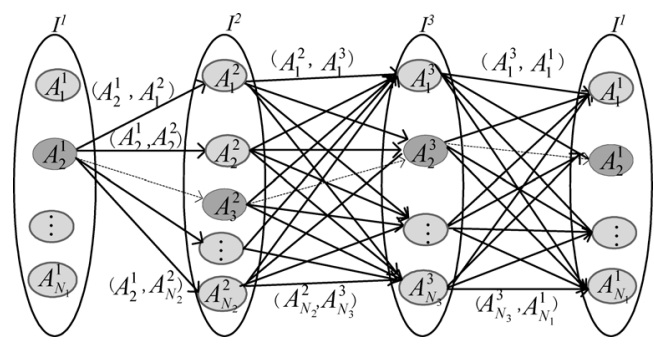

Fig. 3. Negative cycle search using approximately dynamic programming. 
most negative transfer cost, but they cannot form a cycle. We repeat the dynamic programming until all the slabs in the $I^{1}$ have been taken as the starting slab. If there are no negative cost cycles found for a certain permutation, we attempt to simultaneously reduce the cycle's cost by modifying its length. This procedure will be iteratively performed for a given time to improve the solution.

\subsubsection{Restricted CT Neighborhood}

Since the cyclic transfer neighborhood is very large, it would be injudicious to compute each arc cost in the improvement graph for each subset of slabs. Nevertheless, we can identify carefully selected special nodes and search this restricted neighborhood to enhance the algorithm without worsening the time needed to find negative cost cycles. To speed up the algorithm, we use two methods to restrict the neighborhood search. The first method is searching only a restricted subset of the cyclic transfer neighborhood. Therefore, we should not need to search the entire improvement graph. The second method is the slab block exchange.

In the actual production, a large jump in width between adjacent slabs is far worse than a large jump in other factors influencing the hot rolling scheduling, it is desirable that the change in width between adjacent slabs be kept to a minimum. Since the sequence of slabs should be rolled in the width profile of particular coffin shape, it is obvious that some invalid exchanges exist. Figure 4(a) shows illustration of the slab exchange between turns. It is unreasonable that the slab in the section 1 should be exchanged one in the section 2 between turns. Therefore, let us assume that slabs should be exchanged only in the same sections between turns. A reduction technique is adopted for searching only a restricted subset of the CT neighborhood. The use of this technique allows us to ignore redundant operations substantially if we are interested in finding only the valid exchanges.

The reduction technique is a restriction with respect to the number of exchanged slabs between turns. The slab range of each turn is partitioned into $N R^{\prime}$ sections. By attaching a restriction for each section range, a criterion for the selection of exchanged slabs is established. In this study, we set $N R^{\prime}=4$ and prescribe the partition ranges as follows, where $\left[A_{a_{i}^{i}}^{i}, A_{b_{i}^{i}}^{i}\right]$ represents the section $i^{\prime}$ of the turn $i$ and the boundaries are $A_{1}^{i}$ and $A_{N i}^{i}$. Let $N_{R}^{i}$ be the slab number of a section, which is the same in the first three sections, and is different in the last section.

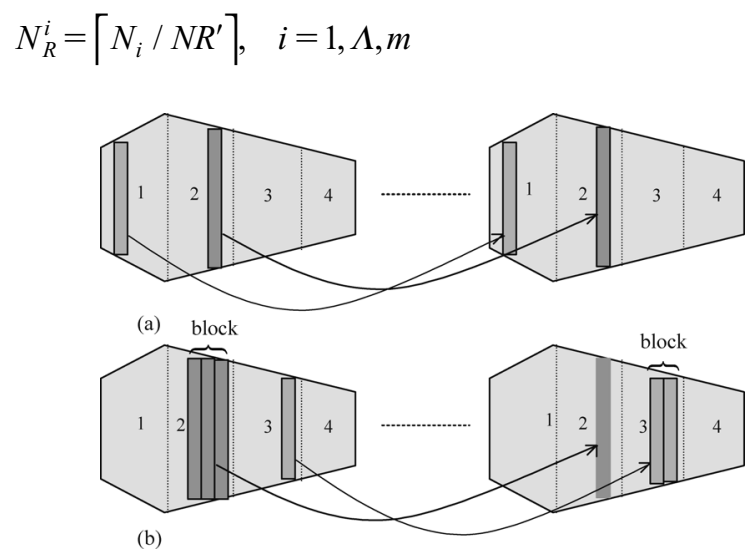

Fig. 4. Illustration of the slab exchange between turns.

$$
\begin{aligned}
& a_{i^{\prime}}^{i}=1+\left(i^{\prime}-1\right) N_{R}^{i}, \quad i^{\prime}=1, \Lambda, N R^{\prime} \\
& b_{i^{\prime}}^{i}= \begin{cases}i^{\prime} N_{R}^{i}, & i^{\prime}=1, \Lambda, N R^{\prime}-1 \\
N_{i}, & i^{\prime}=N R^{\prime}\end{cases}
\end{aligned}
$$

The iteration relationship of the best cost of transfers from the node $A_{2}^{1}$ in turn $\rho(1)$ to every node $j$ in turn $\rho(k+1)$ is:

$$
\begin{gathered}
f_{1}^{i^{\prime}}\left(A_{2}^{1}, A_{j}^{2}\right)=c\left(A_{2}^{1}, A_{j}^{2}\right), j=a_{i^{\prime}}^{2}, \cdots, b_{i^{\prime}}^{2}, i^{\prime}=1, \Lambda, N R^{\prime} \ldots \\
f_{k}^{i^{\prime}}\left(A_{2}^{1}, A_{j}^{k+1}\right)=\min _{a_{i^{k}} \leq i \leq b_{i^{\prime}}}\left\{c\left(A_{i}^{k}, A_{j}^{k+1}\right)+f_{k-1}^{i^{\prime}}\left(A_{2}^{1}, A_{i}^{k}\right)\right\}, \\
\quad j=a_{i^{\prime}}^{k+1}, \cdots, b_{i^{\prime}}^{k+1}, k=2, \cdots, m, i^{\prime}=1, \Lambda, N R^{\prime} \ldots
\end{gathered}
$$

The second method is adopted to reduce the number of the CT neighborhood by means of the slab block exchange. In one turn data, some slabs have similar characteristics such as width, thickness, grade, hardness, etc. If the sum of the penalty of these slabs is less than a predetermined amount in one turn, we consider these slabs as a block, which consists of a small subset of similar characteristic slabs. The slabs are exchanged in the same block simultaneously from one turn to another turn (see Fig. 4(b)). These methods are effective to reduce the computational requirements to reasonable levels in practice.

\subsection{CT_ACO Algorithm}

Ant colony optimization $(\mathrm{ACO})^{9)}$ is a new metaheuristic algorithm and it has been successfully applied to many combinatorial optimization problems, such as $\mathrm{TSP}^{10)}$ and VRP. ${ }^{11)}$ Ant colony optimization includes the two basic steps of construction of solutions and improvement strategies of the solution, when it was adapted to solve the HRSP. It is necessary for one turn to determine a nice sequence of slabs with the least cost. Each individual ant starting at the virtual node simulates a turn and constructs the slab sequence of the turn. Initially, the ant $k$ at the slab $i$ successively selects the next slab $j$ from $A_{2}$ (i.e., $x_{i j k}^{2}=1$ ) and forms the sequence of slabs in the warm up material section until the capacity constraint of the section is met. Then from there the ant at the slab $i$ continues to choose the next slab $j$ from $A_{1}$ (i.e., $\left.x_{i j k}^{1}=1\right)$ and forms the sequence of slabs in the staple material section until the capacity constraint of the staple material section is met. The selected slabs should first satisfy constraints of smooth changes of the adjacent slabs in hardness, temperature and gauge as defined in constraints (12), (13) and (14), and then the rolling length of the consecutive slabs with same width in the staple material section cannot exceed a predetermined amount as described in constraints (6). In addition to these constraints, the selected slabs should satisfy all the other constraints as described in Sec. 2.2. If a slab violates one or more of the constraints, the slab should not be chosen, and the next slab will be selected to check whether the constraint condition is satisfied. If the selected slabs satisfy all the constraints, the ant $k$ at the slab $i$ selects the next slab $j$ to roll based on the following probabilistic formula:

$$
j= \begin{cases}\underset{\mu \notin M_{\kappa}}{\operatorname{argmax}}\left\{\tau_{i u} \cdot\left[\eta_{i u}\right]^{\beta}\right\}, & \text { if } q \leq q_{0} \\ \Gamma, & \text { otherwise }\end{cases}
$$


where $\tau_{i u}$ is the intensity of pheromone trail on edge $(i, u)$, and $\beta$ is a parameter. The value $q$ is a random uniform variable in $[0,1]$, and the value $q_{0}$ is a parameter $\left(0 \leq q_{0} \leq 1\right)$. The visibility $\eta_{i u}$ is defined as the inverse of the penalty $C_{i j}^{l}$. $M_{k}$ is the set of slabs that ant $k$ has already rolled and are not allowed to choose. If $q \leq q_{0}$ then the ant selects the best edge depending on Eq. (25); otherwise an edge is chosen according to $\Gamma$. $\Gamma$ denotes a random variable based on the probabilistic distribution as the following equation:

$$
P_{i j}^{k}= \begin{cases}\frac{\tau_{i j} \cdot\left[\eta_{i j}\right]^{\beta}}{\sum_{u \notin M_{k}} \tau_{i u} \cdot\left[\eta_{i u}\right]^{\beta}}, & j \notin M_{k} \\ 0, & \text { otherwis }\end{cases}
$$

where $p_{i j}^{k}$ gives the probability with which ant $k$ chooses to roll from slab $i$ to slab $j$.

Ants deposit a certain amount of pheromone trail while moving. The pheromone trail is updated both locally and globally. Local updating is performed during solutions construction while global updating is performed after all ants have completed their turns. Local updating is intended to avoid a very strong edge being chosen by all the ants. On the other side, global updating is used to intensify the search in the neighborhood of the best solution. In our ACO algorithm, only the best solution is used to globally modify the pheromone trail. We adopt local and global updating rules presented in Ref. 9).

We proposed a CT_ACO algorithm, which is to hybridize ACO and CT algorithm with heuristics to solve the HRSP. At each iteration, ants probabilistically construct solutions to the problem under consideration. The 2-opt heuristic is applied at all times to act as an improvement heuristic within the turn, while the CT procedure is embedded into $\mathrm{ACO}$ as neighborhood search to improve solutions for the between turns. To save computation time, we restrict the certain frequencies of using the $\mathrm{CT}$ procedure such as at every 10 iterations, or we execute it only if the best solution is improved. Moreover, the experimental results have shown there is no probability of finding negative cost cycles, if the value of cyclic permutation is very higher. So we limit the initial search to small negative costs cycle. These methods can reduce the computation time requirements to reasonable levels. The steps of CT_ACO procedure will be addressed as follows:

Step 1: Initialize the trails and parameters. Allocate $m$ ants to the virtual node.

Step 2: Each ant builds a turn. Perform local pheromone updating.

Step 3: Improve the turn quality by 2-opt heuristic.

Step 4: Repeat Steps 2 and 3 until a solution is generated.

Step 5: Execute CT procedure. Compute arc costs of cyclic transfer and form cost matrix $C^{k}$.

Step 6: Check whether there is the minimum negative cost. If there exists the negative cost cycle, backtrack to all the nodes constituting a cycle and record it. Otherwise, modify the cyclic length, repeat Step 5 until we find the negative cost cycle.

Step 7: Calculate the objective value and check whether the best solution is improved.

Step 8: Update global pheromone trail and record the best solution.

Step 9: Repeat Steps 2-8 until a terminating criterion is met.

\subsection{CT_SS Algorithm}

Scatter search (SS), introduced by Glover in 1977, is a population-based metaheuristic algorithm that combines solutions from a reference set. SS maintains in a reference set some high-quality solutions during the search process and also ensures diversity to explore other regions when the process is trapped in a local minimum. ${ }^{12)} \mathrm{SS}$ has been applied successfully to many combinatorial optimization problems. Marti and Laguna ${ }^{13)}$ and Glover ${ }^{14)}$ provide detailed descriptions and an implementation framework of the SS approach. Russel and Chiang, ${ }^{12)}$ and Greistorfer ${ }^{15)}$ applied SS to the VRP. The SS method is not restricted to a single uniform design, and it is very flexible and effective, since each of its elements can be implemented in variety of ways. Because the HRSP with practical constraints is extremely complex, quality solutions require an elaborate design of the algorithm. Therefore, we proposed a CT_SS algorithm, which is to hybridize CT neighborhood search and SS with heuristics. This method is based on the SS template, which consists of the following elements.

- A reference set generation method. The reference set ${ }^{16)}$ is a set of feasible solutions, so we first should generate it with a greedy heuristic based on simulating the scheduler's manual method. Initially, in the warm up material section, the least width slab chosen from the warm up material set is considered as the first slab of a turn. From there choose the least cost slab not yet rolled, and repeat this process until the capacity constraints of the section is met. Then in the staple material section, on the contrary, the more width and least cost slab adjacent the last slab chosen from the staple material set is considered as the first slab. From there choose the least cost slab not yet rolled, and continue this process until the capacity constraints of turns in the staple material section is met. This procedure continues until the initial solution set is generated. The reference set consists of a total of $b_{1}$ best solutions and $b_{2}$ solutions chosen randomly from the initial solution set.

- A solution combination method. We adopt 2-solution combination method to generated new solutions from in the reference set. Let the reference set $\operatorname{RefSet}=\left\{s_{1}, s_{2}, s_{3}, \ldots\right.$, $\left.s_{|R e f S e t|}\right\}$. 2-solution combination subsets consist of $\left\{s_{1}, s_{2}\right\}$, $\left\{s_{1}, s_{3}\right\}, \ldots,\left\{s_{2}, s_{3}\right\}, \ldots,\left\{s_{|R e f S e t|-1}, s_{|R e f S e t|}\right\}$. To combine solutions from the reference set, common arcs are generated. ${ }^{17)}$ A common arc is shared by each solution of a subset, and it should increase a probability of being chosen. After both turns of different solutions are matched, the common arcs are generated. According to these common arcs, a partial turn can be produced. This procedure is continued until a partial solution can be generated. In special cases, a combination subset may not produce a partial solution if there are a few common arcs. Based on these common arcs, $N_{\text {num }}$ partial solutions are generated. To make partial solutions feasible, a repairing procedure based on the greedy heuristic is then used to connect the remaining slabs. This procedure is mainly used to insert unrolled slabs into turns in the current partial solution. As far as the process goes, $N_{\text {num }}$ complete solutions are generated at the end. 
- A reference set update method. The new reference set consists of the best $[b / 2]-1$ solution from the set of $N_{\text {num }}$ solutions, $[b / 2]-1$ solutions from the current reference set, a solution randomly selected from $N_{\text {num }}$ solutions and a solution generated with a greedy heuristic to extend the diversity of the solutions. The solutions in the reference set are sorted by increasing their objective function values, where the best solution is the first one in the list.

The terminating criterion is the maximum number of iterations. This procedure is repeated until this number is achieved. The steps of the CT_SS procedure are illustrated as follows:

Step 1: Generate initial solutions. Initialize data and create different initial solutions with a greedy heuristic.

Step 2: Build the reference set (RefSet).

Step 3: Find the best solution BestSol in the reference set.

Step 4: Combine RefSet solutions. First, common arcs are assembled into $N_{\text {num }}$ partial solutions, and then $N_{\text {num }}$ partial solutions are changed into $N_{\text {num }}$ complete solutions.

Step 5: Improvement solutions. 2-opt and CT are executed to improve solution quality.

Step 6: Update the reference set. Choose $b$ solutions to update the reference set and compute the objective function values and sort the solutions in the reference set.

Step 7: Update the best solution Bestsol. The Bestsol is updated if the improvement generates a better solution than BestSol.

Step 8: Repeat Steps 4-7 until a stopping criterion is met.

\section{Decision Support System Construction}

We have designed a decision support system for the hot rolling scheduling. The system incorporates the mathematical algorithms, modern information technology and expertise in the hot rolling scheduling, so it not only provides mechanisms to improve planning ability and overall system effectiveness, but also deals with the problem of planning with uncertain information. The emphasis of our designed system is that the mathematical model and solution method have been embedded in the designed decision system. The architecture of the decision support system is shown in Fig. 5. The system consists of many components such as Model and algorithm, Hot rolling scheduling editor, etc. Model and algorithm component is the core of the decision support system. In special cases, Model component can be modified to satisfy practical requirements if there are additional requests from customers. The system can generate better turns in the short time available, and the user-friendly and intuitive graphical user interface allows the planner to evaluate and modify easily the turns manually by analyzing the resulting turns before accepting the turn for production.

Hot rolling scheduling editor was programmed to provide most of the schedule manipulation functions. The decision support system can generate automatically multiple turns for a shift. Then hot rolling scheduling editor also allows schedulers to edit manually outputs. In the graphic display the hot rolling sequence should be shown, and each

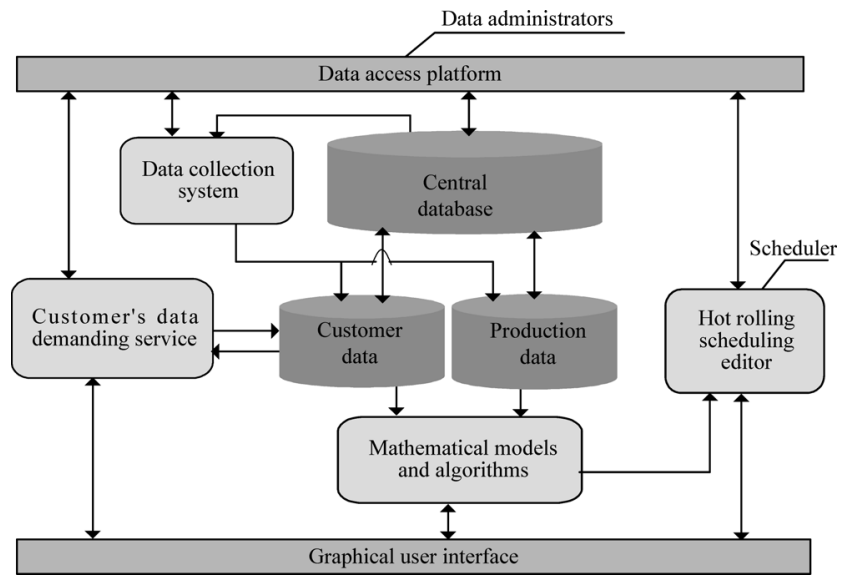

Fig. 5. Decision support system architecture.

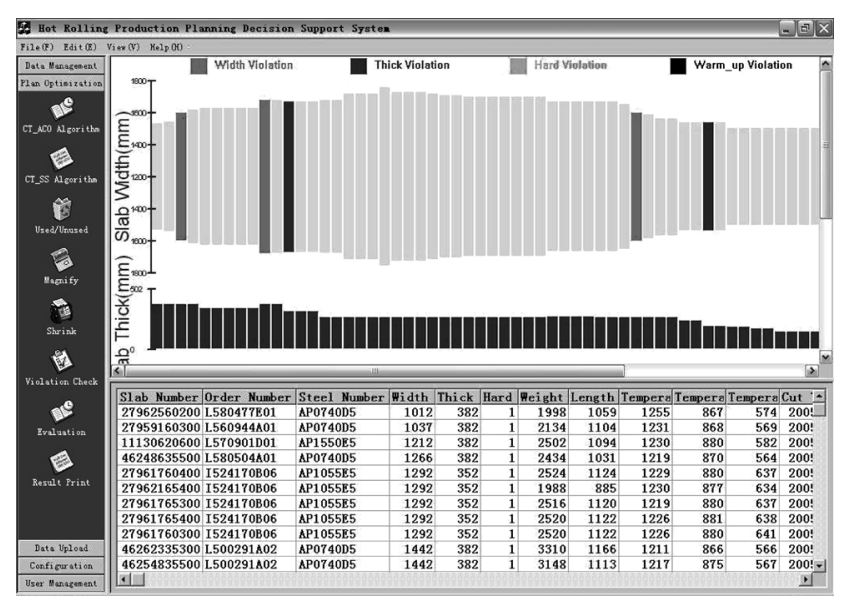

Fig. 6. The graph show of violation check.

slab can be represented by a rectangle symbol whose height is proportional to the slab width. The data display should show the detailed information about slabs in the same sequence. The scheduler can observe clearly the information of each turn and ascertain the performance of the schedule by viewing graphical displays of all slab dimensions. The system can pinpoint where a schedule is deficient, and the scheduler may then edit the turn. Figure 6 shows the graph show of violation check using the different color schemes. Schedulers can edit turns conveniently according to a variety of analyzed geographic and tabulated data. Using the schedule editor, it is easy to create the hot rolling schedule in short time, and the schedule can be edited time and again until a satisfactory new schedule has been obtained.

\section{Computational Results}

In this section, we present computational results with two hybrid algorithms for the HRSP. These algorithms and decision support system was programmed in Microsoft Visual $\mathrm{C}++$, and executed on the IBM computer with $512 \mathrm{MB}$ RAM. The algorithms are tested on problem instances generated randomly and real production data from Shanghai Baoshan Iron and Steel Complex. The objective of the computational experiments is to evaluate the performance of the algorithms in terms of quality of the solutions and computing time. Another aim is to compare the performance of the CT_ACO algorithm and CT_SS method with the current 
scheduling method. Because there are no benchmark instances to test the validity and efficiency of hybrid algorithms for the HRSP, it is difficult to evaluate directly the performances of the proposed algorithms. Therefore, first, we evaluate the performance of two hybrid algorithms on problem instances generated randomly according to production practice situation. Then, we test them on real production data. Finally, two hybrid algorithms are proved to be very efficient compared with the current scheduling method in terms of solution quality.

In general, many parameters in our algorithms may be considered, and these algorithms use the same structure of the penalty. The structure of the penalty function due to changes in width, hardness, gauge and steel grade is proposed according to the practical application of the hot scheduling and the hot rolling operation rules. Many parameters exist in the CT_ACO algorithm, and the values of these affect directly solution quality. In most cases the values have been determined through direct numerical experiments. Experiments have been done with the following parameter settings: $q_{0}=0.95, \beta=6$ and $\rho=0.04$. The objective is a linear combination of four penalty functions and the scheduling objective is to minimize the total penalty cost. Since the product's quality is most important in the four functions, we set $\lambda_{1}>\lambda_{2}, \lambda_{2}>\lambda_{3}$ and $\lambda_{3}>\lambda_{4}$. Let $\lambda_{1}=0.4$, $\lambda_{2}=0.3, \lambda_{3}=0.2$ and $\lambda_{4}=0.1$. Let setup cost $V=500,1000$, the cost of warm up material slabs used in staple material sections $B=80$ and maximum iterations $=1000$. The unrolled slab penalty $\gamma_{i}$ is randomly generated in [50, 150]. In CT_SS algorithm, let $\mid$ RefSet $\mid=5$.

\subsection{Performance of CT_ACO and CT_SS Algorithms}

To evaluate validity of two hybrid algorithms for the HRSP, problem instances were generated randomly according to production practice situation for a shift. There are 60 slabs in every turn and the shift includes six turns. Tables 1 and 2 summarize the results of two algorithms on the problem instances with sizes ranging from 120 to 360 slabs. From the results in Tables 1 and 2, where ACO refers to pure ACO, SS to pure SS, and CT_ACO and CT_SS are the algorithms we proposed, in terms of solution quality, we can see that the minimal penalty and average penalty of solutions obtained by CT_ACO is far less than ones by ACO, and CT_SS performed consistently better than the general SS heuristic. This demonstrates that the cyclic transfer method does improve the quality of the solutions obtained by ACO and SS algorithms, and the average improvements of two algorithms are, respectively, about $3.49 \%$ for CT_ACO and $5.66 \%$ for CT_SS in total penalty. The CT method based on the large-scale neighborhood is capable of exploring the solution space more extensively, so the high-quality solutions may possibly be found. Moreover, CT_ACO generates slightly better quality solutions than CT_SS algorithm. Since the more minimum penalties for the changes between adjacent slabs can deposit higher pheromone, the probability of ants following these more minimum penalties would be higher than that of those following the more maximum penalties. ACO method has the advantages of the ability to find the high-quality feasible solutions in a short time while SS adopts the greedy heuristic based on simulating the scheduler's manual method to
Table 1. The comparison of CT_ACO with ACO algorithm.

\begin{tabular}{|c|c|c|c|c|c|c|c|}
\hline \multirow{3}{*}{ Total slabs } & \multicolumn{3}{|c|}{$\mathrm{ACO}$} & \multicolumn{3}{|c|}{ CT_ACO } & \multirow{3}{*}{$\begin{array}{c}\text { Improvemen } \\
(\%) \\
\end{array}$} \\
\hline & & & & & & & \\
\hline & Min penalty & $\begin{array}{l}\text { Average } \\
\text { penalty }\end{array}$ & Time(s) & Min penalty & $\begin{array}{l}\text { Average } \\
\text { penalty }\end{array}$ & Time(s) & \\
\hline 120 & 6608 & 8130 & 1.20 & 6397 & 7361 & 75 & 3.19 \\
\hline 180 & 8124 & 9675 & 1.52 & 7890 & 8679 & 146 & 2.88 \\
\hline 240 & 10129 & 12382 & 2.59 & 9786 & 12112 & 238 & 3.39 \\
\hline 300 & 11470 & 15497 & 3.74 & 10988 & 15019 & 329 & 4.20 \\
\hline 360 & 11810 & 17770 & 4.01 & 11360 & 16656 & 464 & 3.81 \\
\hline
\end{tabular}

Table 2. The comparison of SS with CT_SS algorithm.

\begin{tabular}{|c|c|c|c|c|c|c|c|}
\hline \multirow{3}{*}{ Total slabs } & \multicolumn{3}{|c|}{ SS } & \multicolumn{3}{|c|}{ CT_SS } & \multirow{3}{*}{$\begin{array}{c}\text { Improvement } \\
(\%)\end{array}$} \\
\hline & \multirow[b]{2}{*}{ Min penalty } & \multirow[b]{2}{*}{$\begin{array}{l}\text { Average } \\
\text { penalty }\end{array}$} & \multirow[b]{2}{*}{ Time(s) } & \multirow[b]{2}{*}{ Min penalty } & \multirow[b]{2}{*}{$\begin{array}{l}\text { Average } \\
\text { penalty }\end{array}$} & \multirow[b]{2}{*}{ Time(s) } & \\
\hline & & & & & & & \\
\hline 120 & 7632 & 9346 & 1.24 & 7151 & 8923 & 83 & 6.30 \\
\hline 180 & 8551 & 10818 & 2.39 & 8036 & 9167 & 187 & 6.02 \\
\hline 240 & 10927 & 12439 & 3.58 & 10145 & 11908 & 305 & 7.16 \\
\hline 300 & 11994 & 14649 & 4.86 & 11327 & 13851 & 381 & 5.56 \\
\hline 360 & 12027 & 16097 & 9.28 & 11638 & 15734 & 534 & 3.23 \\
\hline
\end{tabular}

generate feasible solutions. Therefore, ACO with particular construction mechanism can find the better solutions than $\mathrm{SS}$. The results have proven that the feasible solutions has very influence on the quality of the final solution obtained using cyclic transfer procedure, and the good feasible solutions give significantly better final solutions, i.e., cyclic transfers are better suited to turn improvement than to turn construction. Finally, Tables 1 and 2 have shown that the computation time of CT_ACO and CT_SS is higher than $\mathrm{ACO}$ and SS. This is because, cyclic transfer procedure takes much longer time to construct improvement graphs or find negative cost cycles.

\subsection{Comparison between $C T$ ACO and CT_SS Algo- rithm}

To make sufficient comparisons of hybrid algorithms, we test them on real production data, and some problem results are described in Table 3. Since the ACO method can find the better solutions than SS, and the good feasible solutions give significantly better final solutions obtained using the CT, the CT_ACO algorithm generates slightly better quality solutions than CT_SS algorithm, and the improvement has been made from 1.90 to $3.80 \%$ in total penalty. In this experiment the total penalty of the schedule generated by the CT_ACO method has decreased by 2.8 compared with the result of CT_SS algorithm on average. Furthermore, scatter search is a population-based meta-heuristic which takes much longer time to create improved solutions by combining solutions from the reference set, so the computation speed of CT_SS was slower than that of CT_ACO algorithm, and the results are presented in Table 3. These results also demonstrate that a hot rolling schedule produced using the proposed CT_ACO is more efficient. Moreover, the decision support system can also show automatically the gen- 
Table 3. The comparison of CT_ACO with CT_SS algorithm.

\begin{tabular}{|c|c|c|c|c|c|c|c|}
\hline \multirow{2}{*}{$\begin{array}{l}\text { Total } \\
\text { slabs }\end{array}$} & \multicolumn{3}{|c|}{ CT SS } & \multicolumn{3}{|c|}{ CT_ACO } & aprovement \\
\hline & Min penalty & $\begin{array}{l}\text { Average } \\
\text { penalty }\end{array}$ & Time(s) & Min penalty & $\begin{array}{l}\text { Average } \\
\text { penalty }\end{array}$ & Time(s) & $(\%)$ \\
\hline 122 & 6983 & 9556 & 81 & 6851 & 9467 & 76 & 1.90 \\
\hline 190 & 8544 & 10399 & 191 & 8338 & 9795 & 158 & 2.41 \\
\hline 233 & 9582 & 13777 & 302 & 9274 & 12412 & 233 & 3.21 \\
\hline 291 & 10912 & 15694 & 366 & 10497 & 14649 & 311 & 3.80 \\
\hline 349 & 11919 & 17374 & 521 & 11595 & 16092 & 450 & 2.72 \\
\hline
\end{tabular}

Table 4. The comparison of CT_ACO and CT_SS with the manual scheduling method.

\begin{tabular}{|c|c|c|c|c|c|c|c|}
\hline \multirow{2}{*}{$\begin{array}{l}\text { Total } \\
\text { slabs }\end{array}$} & \multicolumn{2}{|c|}{ CT_ACO } & \multicolumn{2}{|c|}{ CT_SS } & \multirow{2}{*}{$\begin{array}{c}\text { Manual method } \\
\text { Min penalty }\end{array}$} & \multicolumn{2}{|c|}{ Improvement (\% } \\
\hline & Min penalty & Time(s) & Min penalty & Time(s) & & Gapl & Gap2 \\
\hline 122 & 9579 & 50 & 9686 & 79 & 10617 & 9.78 & 8.77 \\
\hline 190 & 12893 & 127 & 13101 & 165 & 16083 & 19.83 & 18.54 \\
\hline 233 & 15223 & 224 & 15636 & 284 & 18605 & 18.18 & 15.96 \\
\hline 291 & 17216 & 296 & 17755 & 358 & 26720 & 35.57 & 33.55 \\
\hline 349 & 20950 & 423 & 21386 & 507 & 28200 & 25.711 & 24.16 \\
\hline
\end{tabular}

erated sequences and the detailed analysis report. According to the information, schedulers may edit manually outputs through an intuitive graphical interface to satisfy customer and production requirements if necessary.

\subsection{Comparison with the Current Scheduling Method}

As described before, two hybrid algorithms can achieve more effective hot rolling scheduling. In order to evaluate the performance of the proposed algorithms compared with current scheduling method, the mathematical model should be simplified. From the model in Sec. 2.3, the lower bound in constraints (4) and constraints (2) can determine that every slab needn't be rolled compulsorily in the slab pool. Therefore, we relax the lower bound in constraints (4) and modify constraints (2) into $\sum_{k=1}^{m} y_{i k}=1, i \in N$, the mathematical model is simplified as the model, where these slabs must be rolled compulsorily in the slab pool. The penalties to be paid for the unrolled slabs should be zero in the objective function. Table 4 reports the comparison of CT_ACO and CT_SS with the current method used to generate actual production schedules. In Table 4, column Gap1 denotes the result of the comparison of CT_ACO with the current scheduling method while column Gap2 denotes the result of the comparison of CT_SS with the current scheduling method.

The following results can be made. 1) In terms of solution quality, the experimental results have shown that two algorithms produce better results than the current scheduling method, but CT_ACO is slightly better quality solutions than CT_SS and more improvement has been made in total penalty compared with the current scheduling method. CT_ACO algorithm can achieve as much as $22 \%$ average improvement, while the average improvements of CT_SS algorithm is about $20 \%$. 2) In terms of the computation time, two hybrid algorithms reduce the time for creating the hot rolling schedules. Two hybrid algorithms take about a few minutes to produce the hot rolling schedules for one shift, while the current scheduling method takes about half an hour.

\section{Conclusions}

In this paper we have studied the HRSP and presented the model characterized by some new features. These features make the solution methodology more difficult. Therefore, two hybrid algorithms are proposed to determine good approximate solutions for this complicated problem. The computational experiments have shown that each hybrid strategy is superior to the general algorithm. Moreover, two hybrid algorithms have more potential for improvement to solve the HRSP compared with the manual scheduling method, but the CT_ACO generates slightly better quality solutions than CT_SS algorithm. Finally, a decision support system for the HRSP is designed to improve the existing scheduling process, and the performance of the system has been tested using various scheduling scenarios. The most popular feature of the system is architecture of component management, which allows us to modify easily some components according to the practice situation so that the decision support system is convenient enough to be applied to other steel companies.

\section{Acknowledgements}

This research is partly supported by National Natural Science Foundation for Distinguished Young Scholars of China (Grant No. 70425003), National 863 High-Tech Research and Development Program of China through approved No. 2006AA04Z174 and National Natural Science Foundation of China (Grant No. 60674084).

\section{REFERENCES}

1) L. Lopez, M. W. Carter and M. Gendreau: Eur. J. Oper. Res., 106 (1998), 317.

2) P. Cowling: Optimisation in Steel Hot Rolling, Optimization in Industry, Wiley, Chichester, England, (1995), 55.

3) P. Cowling and W. Rezig: J. Scheduling, 3 (2000), 185.

4) E. Balas: Networks, 19 (1989), 621.

5) L. X. Tang, J. Y. Liu, A. Y. Rong and Z. H. Yang: Eur. J. Oper. Res., 124 (2000), 267.

6) P. Cowling: Computers and Industrial Engineering, 45 (2003), No. 2, 307.

7) P. M. Thompson and J. B. Orlin: The Theory Cyclic Transfers, Working Paper, Operations Research Center, MIT, (1989).

8) P. M. Thompson and H. N. Psaraftis: Oper. Res., 41 (1993), No. 5, 935 .

9) J. E. Bell and P. R. McMullen: Adv. Eng. Informat., 18 (2004), 41.

10) M. Dorigo and L. M. Gambardella: BioSystems, 43 (1997), 73.

11) B. Bulleneimer, R. F. Hartl and C. Strauss: Ann. Oper. Res., 89 (1999), 319.

12) R. A. Russell and W. C. Chiang: Eur. J. Oper. Res., 169 (2006), 606.

13) M. Marti, M. Laguna and F. Glover: Eur. J. Oper. Res., 169 (2006), 359.

14) F. Glover: Lecture Notes in Computer Science, 1363 (1998), 13.

15) P. Greistorfer: Computers \& Industrial Engineering, 44 (2003), No. $2,249$.

16) A. Corberan, E. Fernandez, M. Laguna and M. Marti: J. Oper. Res. Soc., 53 (2002), No. 4, 427

17) J. P. Kelly and J. Xu: INFORMS, 11 (1999), No. 2, 161. 